\title{
The Abdominal Aortic Intima-Media Thickness Increases in Patients with Primary Hyperparathyroidism
}

Authors

Hilmi Erdem Sumbul1', Ayse Selcan Koc²

\section{Affiliations \\ 1 Department of Internal Medicine, University of Health Sciences - Adana Health Practice and Research Center, Adana, Turkey \\ 2 Department of Radiology, University of Health Sciences - Adana Health Practice and Research Center, Adana, Turkey}

\begin{abstract}
Key words
primary hyperparathyroidism, aortic intima-media thickness, parathyroid hormone
\end{abstract}

$\begin{array}{ll}\text { received } & 06.06 .2018 \\ \text { revised } & 05.07 .2018 \\ \text { accepted } & 26.07 .2018\end{array}$

\author{
Bibliography \\ DOI https://doi.org/10.1055/a-0664-7820 \\ Published online: 14.8.2018 \\ Exp Clin Endocrinol Diabetes 2019; 127: 405-412 \\ (C) J. A. Barth Verlag in Georg Thieme Verlag KG Stuttgart . \\ New York \\ ISSN 0947-7349 \\ Correspondence \\ Koc Ayse Selcan \\ Department of Radiology \\ University of Health Sciences - Adana Health Practices and \\ Research Center \\ Adana \\ Turkey \\ Tel.: +90/506/2425988 \\ drayseselcankoc@gmail.com
}

\begin{abstract}
Background We aimed to determine whether there is an increase in aortic intima-media thickness values measured from the abdominal aorta in addition to the traditional carotid intima-media thickness in patients with primary hyperparathyroidism and to determine the parameters closely related to aortic intima-media thickness.

Methods This perspective study included 65 primary hyperparathyroidism patients and 30 healthy-controls. Routine laboratory tests for the diagnosis of hyperparathyroidism and vascular ultrasound examinations were performed. Common carotid, internal carotid, and abdominal aortic intima media thickness were measured. The participants of the study was divided into 3 groups as the control (Group-I), the medical treatment (Group-II) due to primary hyperparathyroidism, and the planned surgery (group-III).

Results Aortic intima-media thickness, serum urea, creatinine, glucose, uric acid, hs-CRP, parathyroid hormone, calcium and urine calcium levels increased significantly from Group-I to Group-III and T-scores and serum phosphorus in levels decreased significantly. Carotid intima-media thicknesses were not significantly different between the groups. Serum and urinary calcium levels were independently associated with aortic intima-media thickness. Aortic intima-media thickness, serum phosphorus, parathyroid hormone, T-scores, serum and urinary calcium levels are independent indicators for Group-III. When the cut-off value of aortic intima-media thickness was taken as $1.5 \mathrm{~mm}, 80.6 \%$ sensitivity and $89.1 \%$ specificity were determined for patients who would go to surgery.

Conclusion Serum and urine calcium levels were independently associated with aortic intima-media thickness. Aortic intima-media thickness is more useful than carotid intimamedia thickness in showing vascular organ involvement in patients with primary hyperparathyroidism.
\end{abstract}

\section{Introduction}

Atherosclerosis occurs in the tunica intima and media layers of the vessel wall and causes an increase in intima-media thickness (IMT) in the early period of the disease. IMT can be measured clearly by ultrasonography (USG). In adult and pediatric patients IMT evaluation is performed from carotid artery and abdominal aorta (C-IMT and A-IMT). Besides atherosclerosis, vascular IMT measurement is used for target organ damage detection in many systemic diseases.
Primary hyperparathyroidism (PHPT) is an endocrine disorder associated with long-term subclinical elevated parathyroid hormone (PTH) and mineral abnormalities [1]. Increased levels of calcium and PTH in this disease have been shown to negatively affect vascular function, especially by impairing vasodilation [2]. In the literature, there are conflicting results about the effects on C-IMT in patients with PHPT. In some of these studies it has been shown that C-IMT has been increased [3-6], but no significant increase in 
C-IMT has been found in the other significant part of the studies [7-11].

The abdominal aorta is exposed to shear stress due to its size and branching, and according to studies performed, is the first vascular site to develop atherosclerosis. Therefore, abdominal A-IMT measurement can be used to detect the development of early atherosclerosis [12]. There is no study in literature on A-IMT evaluation obtained from abdominal aorta in adult PHPT patients. For this reason, the importance of A-IMT in patients with PHPT is unknown; it is not a routine examination and is not used. For this reason, we also hypothesized that in patients with PHPT, the accumulation of calcium and the IMT increase due to elevated PTH may be earlier and more prominent in A-IMT than C-IMT. In our study, we aimed to determine whether there is an increase in A-IMT values measured from the abdominal aorta in addition to the traditional C-IMT in patients with PHPT and to determine the parameters closely related to A-IMT.

\section{Materials and Methods}

\section{Study population}

This prospective study included 65 patients (mean age: $57.4 \pm 12.8$ years, male/female: 9/56) biochemically diagnosed with PHPT and 30 healthy controls with normal serum PTH, calcium and phosphate levels (mean age: $56.9 \pm 9.8$ years, male/female:10/20). In the control and PHPT group, care was taken to avoid any other major cardiovascular (CV) risk factors that may increase IMT such as hypertension (HT), smoking, diabetes mellitus (DM), hyperlipidemia (HL). The participants of the study was divided into 3 groups as the control or Group I (healthy-controls), the medical treatment or Group II (patients with asymptomatic PHPT and no surgery indications), and the planned surgery or group III (patients with symptomatic PHPT and asymptomatic PHPT who had to surgery indications). According to the National Institutes of Health consensus panel as the surgical criteria for PHPT patients, symptomatic PHPT patients and asymptomatic PHPT patients who has (i) the serum calcium value exceeds the reference value by $1 \mathrm{mg} / \mathrm{dl}$, (ii) significant hypercalciuria ( $>400 \mathrm{mg} / 24 \mathrm{~h}$ ), (iii) in dual $x$-ray absorptiometry (DEXA) the T score is $<-2.5$, (iv) younger than 50 years $(<50$ years) (v) more than $30 \%$ reduction of creatinine clearance without any cause [13]. Patients with alcohol addiction, cerebrovascular disease, secondary or malignant $\mathrm{HT}$, abdominal aortic aneurysm or dissection, inflammatory diseases, hematologic diseases, coronary artery disease, peripheral vascular disease, congestive heart failure, severe heart valve dysfunction, smoking, obesity, HT, DM and HL diseases, cancer, pregnancy or kidney failure were excluded from both groups. While identifying HT and HL patients, ESC / EAS 2016 hyperlipidemia and ESC 2013 HT guidelines were used and the presence or absence of $\mathrm{HL}$ and $\mathrm{HT}$ were determined according to the limit values in these guidelines $[14,15]$. The Local Ethics Committee approved the study protocol and each participant gave written informed consent.

After a detailed medical history and a complete physical examination, baseline characteristics (age, sex, pulse rate, systolic and diastolic blood pressure) of all groups were recorded. Body mass index was calculated by measuring weight and height. Then rou- tine laboratory tests and vascular USG examinations were performed on all patients. All patients were evaluated for renal stones or nephrocalcinosis in renal USG for surgical indications. Also the bone mineral density was measured on anterior-posterior and lateral lumbar vertebra (L1-L4) in all the patients. The measurements were performed with DEXA (Lunar iDXA, GE, Madison, WI, USA). DEXA was not performed because there was no indication in the control group.

\section{Biochemical measurements}

Serum PTH concentration and 25-hydroxyvitamine D [25(OH) Vit D] were measured using chemiluminescence immunoassay and Beckman Coulter DXI800. Complete blood count was measured using a Beckman Coulter DXH 800 . Serum glucose, HbA1c, total cholesterol, triglyceride, low-density lipoprotein, high-density lipoprotein, hs-CRP, uric acid, blood urea nitrogen (BUN), creatinine, calcium, phosphorus and urine calcium levels were measured using an automated chemistry analyzer (Abbott Aeroset, MN, USA) and using appropriate commercial kits (Abbott). The corrected serum calcium levels were calculated using the most commonly used formula in clinical practice, if serum albumin level was lower than $4 \mathrm{mg} / \mathrm{dl}$ : Corrected calcium $=$ measured total calcium $(\mathrm{mg} / \mathrm{dL})+0.8$ (4.0 - serum albumin [g/dL]) [16].

\section{Common carotid, internal carotid and abdominal aortic B-mode ultrasonography evaluation}

The abdominal aorta and left and right common and internal carotid (CC and IC) arteries were examined with a high-resolution ultrasound system (Philips EPIQ 7) equipped with a $12 \mathrm{MHz}$ linear and $5 \mathrm{MHz}$ convex transducer (Philips Health Care, Bothell, WA, USA). All arteries were examined both longitudinally and transversely. All arteries were scanned longitudinally to visualize the IMT on the posterior or distal wall of the artery. All measurements were made on frozen images. Two images of the best quality were selected for analysis on each study. IMT was defined as the distance from the anterior margin of the first echogenic line to the anterior margin of the second line. The first line represents the intima-lumen interface and the second line represents the collagen-containing top layer of adventitia. Vascular IMT was measured using ultrasonic calipers in the presence of two independent and blind observers. All IMT values were calculated as averages of six measurements.

The patients were examined in the supine position. Patients rotated their heads by $45^{\circ}$ from where they were scanned for the examination of the carotid arteries. CC-IMT and IC-IMT were accepted as IMTs measured from $10-20 \mathrm{~mm}$ proximal before bifurcation (for the main carotid artery) and at the distal segment of the right and left main carotid artery in the distal segment after bifurcation (for the IC artery) respectively. Abdominal A-IMT was investigated at the level ranging from renal artery bifurcation to iliac artery bifurcation. IMT measured from the posterior wall of the abdominal artery was accepted as A-IMT ( $\triangleright$ Fig. 1, 2).

\section{Statistical analysis}

The variables were divided into two as categorical and continuous groups. Continuous variables were expressed as mean \pm standard deviation (mean $\pm S D$ ). Categorical variables were given as numbers and percentages. Comparisons of continuous variables were 


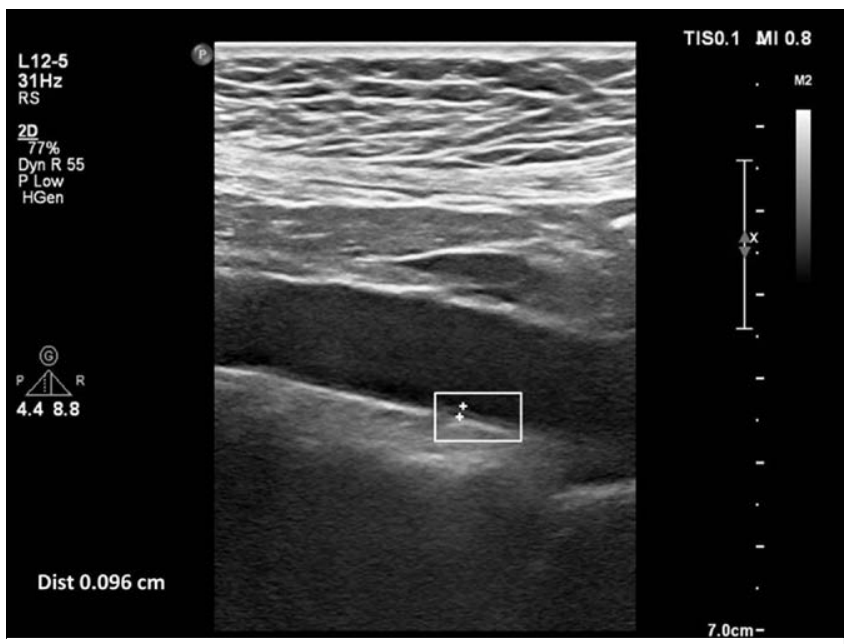

- Fig. 1 B-mode ultrasound image of a patient without primary hyperparathyroidism: normal intima-media thickness on posterior wall of abdominal aorta.

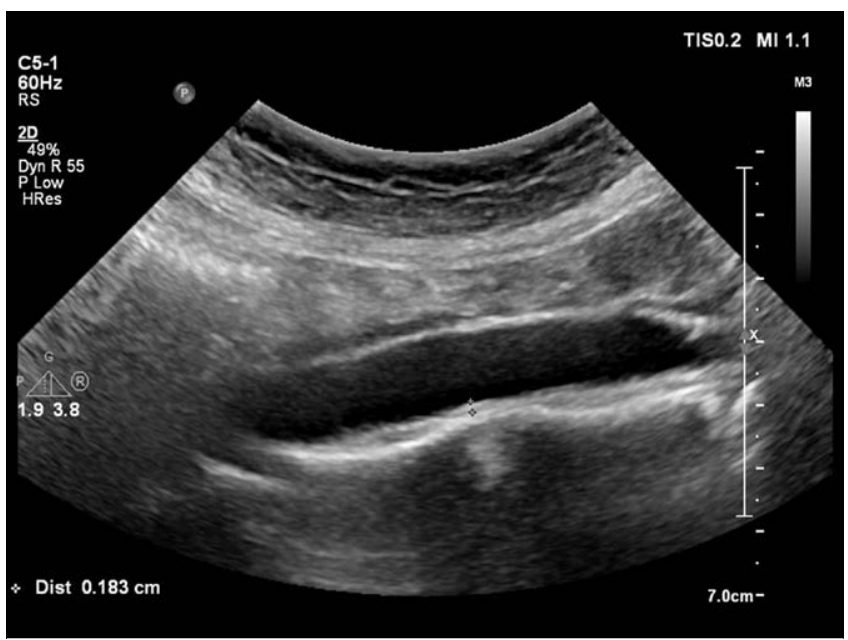

-Fig. 2 B-mode ultrasound image of a patient with primary hyperparathyroidism: increased intima-media thickness on posterior wall of abdominal aorta.

performed by One way ANOVA or Kruskal-Wallis 1-way ANOVA tests according to the distribution. For normally distributed data, Scheffe and Games-Howell tests were used for multiple comparisons of groups with respect to homogeneity of variances. For non-normally distributed data, Bonferroni adjusted Mann Whitney U test was used for multiple comparisons of groups. Chi Square Test was used to compare categorical variables. For independent determination of patients who underwent surgery, multivariate logistic regression analysis was performed. ROC curve analysis was performed to reassess markers that independent for identifying patients to be surgically treated and to determine the border value of these markers. The value of the area under the curve was used as the accuracy criterion of the test. Univariate correlation analysis was performed using Pearson's correlation method to determine the parameters associated with A-IMT. Linear regression analysis is performed with these parameters and independent indicators affecting A-IMT have been identified. Statistical significance level was accepted as $p<0.05$. All analyzes were performed with SPSS 20.0 (Chicago, IL, USA) statistical software package.

\section{Results}

The participants of the study was divided into 3 groups as the control (Group I), the medical treatment (Group II) due to PHPT, and the planned surgery (group III). In our study we found that 8 of the PHPT patients (12.3\%) had renal stones. There was also no fracture in the PHPT patient group in the study.

\section{Demographic, clinical and laboratory findings of the study groups}

There was no difference between groups in terms of general demographic and clinical parameters. BUN, creatinine and uric acid levels were highest in Group III and statistical significance was found only between Group I and Group III ( hs-CRP levels were significantly higher in Group II and Group III compared to Group I ( Table 1). Serum PTH, serum and urine calcium levels increased significantly from Group I to Group III and serum phosphorus levels decreased significantly ( $\vee$ Table 1 ). It was determined that serum PTH, calcium, phosphorus and urine calcium levels were statistically different between all study groups ( $\triangleright$ Table 1). Negatively T scores in DEXA and the presence of $T$ scores in DEXA $<-2.5$ were significantly higher Group III than Group I ( Table 1). Other laboratory findings were similar among the groups ( $\triangleright$ Table $\mathbf{1}$ ).

\section{B-mode ultrasonography findings of study groups}

When CC-IMT and IC-IMT measurements were compared between groups, there was no statistically significant difference between the groups, but there was an increase in CC-IMT and IC-IMT values from Group I to Group III ( $\$$ Table 2 ). The A-IMT value increased significantly from Group I to Group III and it was found that A-IMT level was statistically significantly different among all study groups ( Table 2).

\section{Multivariate logistic regression analysis for the detection of patients treated surgically for primary hyperparathyroidism}

In multivariate logistic regression analysis, it was found that A-IMT, phosphorus, PTH, T scores in DEXA, serum and urine calcium levels independently determined the patients to be treated surgically ( $p<0.05$ and $>$ Table 3). According to this analysis, increased A-IMT (per 0.1), PTH (per $10 \mathrm{pg} / \mathrm{mL}$ ), T scores in DEXA (per 0.2), serum calcium (per $0.1 \mathrm{mg} / \mathrm{dL}$ ), urinary calcium (per $10 \mathrm{mg} / \mathrm{dL}$ ) and decreased serum phosphorus (per $0.2 \mathrm{mg} / \mathrm{dL}$ ) levels were found to increase the risk of patients to undergo surgical treatment by $40.5 \%$, $8.9 \%, 63.0 \%, 29.5 \%, 12.9 \%$ and $27.5 \%$ respectively ( $>$ Table 3 )

\section{ROC analysis for the detection of patients treated surgically for primary hyperparathyroidism}

In the ROC analysis, the area under the curve was $0.901,0.874$, $0.662,0.836,0.797$ and 0.834 for urine calcium, serum calcium, $T$ scores in DEXA, PTH, serum phosphorus and A-IMT respectively $(p<0.05$, $>$ Table 4 and $>$ Fig. 3 ). When the A-IMT and urine calci- 
- Table 1 Demographic, clinical and laboratory findings of the study groups.

\begin{tabular}{|c|c|c|c|c|}
\hline Variable & $\begin{array}{l}\text { Group I } \\
\mathbf{n}=\mathbf{3 0}\end{array}$ & $\begin{array}{l}\text { Group II } \\
n=34\end{array}$ & $\begin{array}{l}\text { Group III } \\
\mathrm{n}=\mathbf{3 1}\end{array}$ & $\mathbf{p}$ \\
\hline Age (year) & $56.9 \pm 9.8$ & $57.9 \pm 7.1$ & $56.8 \pm 15.2$ & 0.895 \\
\hline Gender (female) & 20 & 32 & 24 & 0.308 \\
\hline Systolic blood pressure $(\mathrm{mmHg})$ & $121.9 \pm 14.2$ & $122.4 \pm 12.4$ & $126.5 \pm 16.6$ & 0.495 \\
\hline Diastolic blood pressure $(\mathrm{mmHg})$ & $75.1 \pm 9.2$ & $76.5 \pm 10.2$ & $78.7 \pm 11.2$ & 0.368 \\
\hline Pulse (pulse/minute) & $78.1 \pm 10.4$ & $77.1 \pm 9.8$ & $80.4 \pm 12.1$ & 0.545 \\
\hline Body mass index $\left(\mathrm{kg} / \mathrm{m}^{2}\right)$ & $26.1 \pm 3.7$ & $26.2 \pm 3.5$ & $27.2 \pm 3.5$ & 0.204 \\
\hline White blood cell $(\mu \mathrm{L})$ & $6.50 \pm 1.57$ & $6.92 \pm 1.19$ & $6.66 \pm 1.41$ & 0.465 \\
\hline Hematocrit (\%) & $40.2 \pm 2.9$ & $41.3 \pm 2.1$ & $40.7 \pm 4.3$ & 0.298 \\
\hline Platelet (K/mm3) & $281 \pm 33$ & $315 \pm 131$ & $270 \pm 90$ & 0.135 \\
\hline Glucose (mg/dL) & $89.3 \pm 6.0^{\alpha, \beta}$ & $98.5 \pm 10.3$ & $99.2 \pm 12.4$ & 0.001 \\
\hline Blood urea nitrogen $(\mathrm{mg} / \mathrm{dL})$ & $26.2 \pm 4.6^{\alpha}$ & $27.9 \pm 8.8$ & $33.7 \pm 11.8$ & 0.004 \\
\hline Creatinine (mg/dL) & $0.58 \pm 0.08^{\alpha}$ & $0.61 \pm 0.12^{*}$ & $0.92 \pm 0.87$ & 0.013 \\
\hline Total Cholesterol (mg/dL) & $201 \pm 32$ & $202 \pm 36$ & $194 \pm 54$ & 0.496 \\
\hline Low density lipoprotein (mg/dL) & $125 \pm 14$ & $132 \pm 23$ & $123 \pm 32$ & 0.346 \\
\hline High density lipoprotein (mg/dL) & $45.9 \pm 10.2$ & $48.5 \pm 10.9$ & $47.9 \pm 20.2$ & 0.690 \\
\hline Triglyceride (mg/dL) & $174 \pm 54$ & $171 \pm 46$ & $157 \pm 38$ & 0.795 \\
\hline HbA1c (\%) & $5.41 \pm 0.24$ & $5.58 \pm 0.44$ & $5.65 \pm 0.21$ & 0.006 \\
\hline hs-CRP (mg/L) & $0.21 \pm 0.10^{\alpha, \beta}$ & $0.43 \pm 0.34$ & $0.47 \pm 0.38$ & 0.001 \\
\hline Uric acid (mg/dL) & $4.35 \pm 0.82 \alpha$ & $4.91 \pm 1.11$ & $5.54 \pm 1.25$ & $<0.001$ \\
\hline Serum calcium (mg/dL) & $9.2 \pm 0.35 \alpha, \beta$ & $10.7 \pm 0.55^{*}$ & $11.4 \pm 0.88$ & $<0.001$ \\
\hline Urine calcium (mg/24h) & $37.7 \pm 7.4^{\alpha, \beta}$ & $188 \pm 103^{*}$ & $375 \pm 183$ & $<0.001$ \\
\hline Serum phosphorus (mg/dL) & $3.55 \pm 0.52 \alpha, \beta$ & $3.07 \pm 0.60^{*}$ & $2.64 \pm 0.49$ & $<0.001$ \\
\hline Parathyroid hormone $(\mathrm{pg} / \mathrm{mL})$ & $52.2 \pm 12.6^{\alpha, \beta}$ & $178.5 \pm 103^{*}$ & $358.7 \pm 360.8$ & $<0.001$ \\
\hline $25(\mathrm{OH})$ Vit D (ng/mL) & $18.5 \pm 5.1$ & $16.9 \pm 6.6$ & $17.6 \pm 12.1$ & 0.757 \\
\hline $25(\mathrm{OH})$ Vit $\mathrm{D}<30 \mathrm{ng} / \mathrm{mL} \mathrm{n},(\%)$ & $21(70 \%)$ & $29(85 \%)$ & $24(77 \%)$ & 0.674 \\
\hline T scores in DEXA & - & $-1.69 \pm 0.60$ & $-2.19 \pm 0.86$ & 0.008 \\
\hline T scores in DEXA< $<2.5 \mathrm{n},(\%)$ & - & $3(9 \%)$ & $11(36 \%)$ & 0.014 \\
\hline \multicolumn{5}{|c|}{$\begin{array}{l}\text { The values were shown as mean } \pm \text { standard deviation or } n(\%) \text {, Group I = Control group, Group II = Medical treatment group and Group III = Planed } \\
\text { surgery group, } 25(\mathrm{OH}) \text { Vit D: } 25 \text {-hydroxyvitamine D, DEXA: dual } x \text {-ray absorptiometry; } \alpha=\text { the significant association between the Group I and Group } \\
\text { III }(p<0.05) ; \beta=\text { the significant association between the Group I and Group II }(p<0.05) ; ¥=\text { the significant association between the Group II and Group } \\
\text { III } p<0.05)\end{array}$} \\
\hline
\end{tabular}

um cut-off values were taken as $1.5 \mathrm{~mm}$ and $400 \mathrm{mg} /$ day, respectively, it determines patients to undergo surgery with $80.6 \%$ sensitivity and $89.1 \%$ specificity, and $67.7 \%$ sensitivity and $100 \%$ specificity, respectively ( Table 4). In group I, II and III, 4 (13\%), 9 (27\%) and $25(80 \%)$ patients had A-IMT value $\geq 1.5 \mathrm{~mm}$ respectively. When the ROC curve analysis was performed to determine the group II patients of A-IMT value between Group I and Group II, the area under ROC curve was $0.652(\mathrm{Cl} 0.519-0.786, \mathrm{p}=0.036)$, When the cut-off value was taken as $1.25 \mathrm{~mm}$ for the A-IMT value, it was found that Group II was determined with $61 \%$ sensitivity and $70 \%$ specificity.

\section{Parameters associated with abdominal aortic intima- media thickness}

Correlation analysis was performed between A-IMT and other demographic, clinical and laboratory parameters ( $>$ Table 5). Linear regression analysis was performed using parameters that correlated significantly with A-IMT ( $\triangleright$ Table 5). Serum and urinary calcium levels, and C-IMT were independently associated with A-IMT
( $\triangleright$ Table 5). The relationship between A-IMT and urinary calcium level is shown in > Fig. 4.

\section{Discussion}

The main result of this study is that A-IMT is found to be significantly higher in PHPT patients than in control group. Another striking finding is that with symptomatic PHPT and asymptomatic PHPT who had surgery indications, had increased A-IMT than patients with asymptomatic PHPT and had no surgery indications. In addition, we found that serum and urine calcium levels were the most closely related parameters with A-IMT.

There are conflicting results in the literature regarding the increase of C-IMT in patients with PHPT. C-IMT has been associated with an increase in PHPT patients [3-6], but especially in studies conducted in recent years, there is information that there is no IMT increase in PHPT patients [7-11]. The most important reason for this is that patients are diagnosed in asymptomatic or mild hypercalcemia periods of the disease with routine biochemical tests or 
- Table 2 B-mode ultrasonography findings of study groups.

\begin{tabular}{|c|c|c|c|c|}
\hline Variable & $\begin{array}{l}\text { Group I } \\
n=30\end{array}$ & $\begin{array}{l}\text { Group II } \\
n=34\end{array}$ & $\begin{array}{l}\text { Group III } \\
\mathrm{n}=31\end{array}$ & $\mathbf{p}$ \\
\hline Common carotid IMT (mm) & $0.74 \pm 0.08$ & $0.75 \pm 0.14$ & $0.81 \pm 0.15$ & 0.079 \\
\hline Internal carotid IMT (mm) & $0.64 \pm 0.09$ & $0.68 \pm 0.17$ & $0.71 \pm 0.16$ & 0.169 \\
\hline Abdominal aort IMT (mm) & $1.24 \pm 0.18^{\alpha, \beta}$ & $1.40 \pm 0.30^{*}$ & $1.65 \pm 0.26$ & $<0.001$ \\
\hline
\end{tabular}

- Table 3 Variable regression analysis for the detection of patients treated surgically for PHPT.

\begin{tabular}{|c|c|c|c|}
\hline Variable & Odds Ratio & 95\% Confidence Interval & $\mathbf{p}$ \\
\hline Urine calcium $(10 \mathrm{mg} / 24 \mathrm{~h})$ & 1.129 & $1.076-1.185$ & $<0.001$ \\
\hline Abdominal aort IMT $(0.1 \mathrm{~mm})$ & 1.405 & $1.130-1.747$ & 0.002 \\
\hline Serum phosphorus $(0.2 \mathrm{mg} / \mathrm{dL})$ & 0.725 & $0.565-0.931$ & 0.012 \\
\hline Parathyroid hormone (10 pg/mL) & 1.089 & $1.002-1.184$ & 0.045 \\
\hline Serum calcium $(0.1 \mathrm{mg} / \mathrm{dL})$ & 1.295 & $1.024-1.638$ & 0.031 \\
\hline T scores in DEXA (0.2) & 1.630 & $1.050-2.529$ & 0.029 \\
\hline
\end{tabular}

- Table 4 ROC analysis for the detection of patients treated surgically for PHPT.

\begin{tabular}{|c|c|c|c|c|c|}
\hline Variable & AUROC Curve & $\mathbf{p}$ & Cut-off & Sensitivity & Specificity \\
\hline Urine calcium & $0.901(0.841-0.962)$ & $<0.001$ & $\begin{array}{l}400 \mathrm{mg} / \text { day } \\
300 \mathrm{mg} / \text { day }\end{array}$ & $\begin{array}{l}67.7 \% \\
80.6 \%\end{array}$ & $\begin{array}{l}100 \% \\
84.4 \%\end{array}$ \\
\hline Serum calcium & $0.874(0.795-0.952)$ & $<0.001$ & $\begin{array}{l}11.2 \mathrm{mg} / \mathrm{dL} \\
10.2 \mathrm{mg} / \mathrm{dL}\end{array}$ & $\begin{array}{l}66.5 \% \\
90.3 \%\end{array}$ & $\begin{array}{l}93.7 \% \\
71.9 \%\end{array}$ \\
\hline $\mathrm{T}$ - scores in DEXA & $0.662(0.795-0.952)$ & 0.025 & $-2.5-1.0$ & $\begin{array}{l}61.3 \% \\
93.5 \%\end{array}$ & $\begin{array}{l}91.2 \% \\
67.6 \%\end{array}$ \\
\hline Parathyroid hormone & $0.836(0.758-0.915)$ & $<0.001$ & $\begin{array}{l}170 \mathrm{pg} / \mathrm{mL} \\
110 \mathrm{pg} / \mathrm{mL}\end{array}$ & $\begin{array}{l}77.4 \% \\
87.1 \%\end{array}$ & $\begin{array}{l}79.7 \% \\
64.1 \%\end{array}$ \\
\hline Serum phosphorus & $0.797(0.702-0.892)$ & $<0.001$ & $3.0 \mathrm{mg} / \mathrm{dL} 2.5 \mathrm{mg} / \mathrm{dL}$ & $\begin{array}{l}87.1 \% \\
54.8 \%\end{array}$ & $\begin{array}{l}73.4 \% \\
90.6 \%\end{array}$ \\
\hline Abdominal aort IMT & $0.834(0.743-0.925)$ & 0.001 & $\begin{array}{l}1.5 \mathrm{~mm} \\
1.3 \mathrm{~mm}\end{array}$ & $\begin{array}{l}80.6 \% \\
90.3 \%\end{array}$ & $\begin{array}{l}89.1 \% \\
60.9 \%\end{array}$ \\
\hline
\end{tabular}

- Table 5 The parameters associated with A-IMT and linear regression analysis for parameters significantly correlated with A-IMT.

\begin{tabular}{|c|c|c|c|c|}
\hline \multirow[t]{2}{*}{ Variable } & \multicolumn{2}{|c|}{ Univariate analyze } & \multicolumn{2}{|c|}{ Multivariate analyze } \\
\hline & $\mathbf{p}$ & $\mathbf{r}$ & $\mathbf{p}$ & $\beta$ \\
\hline Age (years) & 0.010 & 0.264 & 0.177 & 0.148 \\
\hline Common carotid IMT (mm) & $<0.001$ & 0.473 & $<0.001$ & 0.473 \\
\hline Internal carotid IMT (mm) & 0.003 & 0.306 & 0.787 & 0.036 \\
\hline Blood urea nitrogen $(\mathrm{mg} / \mathrm{dL})$ & 0.002 & 0.320 & 0.181 & 0.172 \\
\hline 25-hydroxyvitamine D (ng/ml) & 0.049 & 0.225 & 0.070 & 0.188 \\
\hline T scores in DEXA & 0.046 & -0.239 & 0.148 & 0.181 \\
\hline Parathyroid hormone (pg/ml) & 0.008 & 0.269 & 0.065 & 0.192 \\
\hline Serum calcium (mg/dL) & $<0.001$ & 0.521 & 0.028 & 0.253 \\
\hline Urine calcium (mg/24 h) & $<0.001$ & 0.463 & $<0.001$ & 0.390 \\
\hline
\end{tabular}




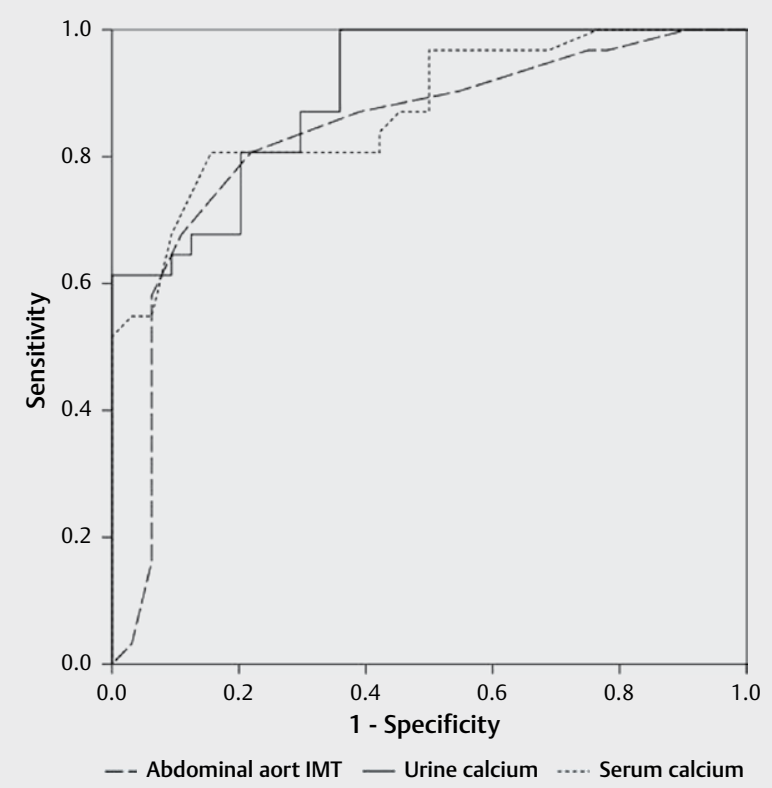

-Fig. 3 The ROC curve of values for abdominal aortic intima-media thickness (IMT), serum and urinary calcium levels for determining patients to be operated for primary hyperparathyroidism.

diagnosed incidentally, and because asymptomatic patients or patients without osteoporosis or nephrolithiasis are treated early with medical and surgical treatments $[13,17]$. The reason for the lack of C-IMT increase in some studies may be that the patients with PHPT may have diagnosed earlier and by early treatment of these patients, high PTH and calcium levels could not affect IMT severely and for a long time. Furthermore, in conducted studies, duration and severity of PHPT disease are different, and often examining only single anatomic region (common or internal carotid IMT) may have caused these different outcomes.

Abdominal A-IMT is affected by systemic diseases earlier than other vascular regions. Therefore, A-IMT may be a sign of earlier involvement in PHPT patients. We have not been able to find any studies investigating the relationship between A-IMT and PHPT patients in literature. In our study, it was determined that A-IMT was increased without a C-IMT increase in PHPT patients. We also evaluated patients with mild PHPT as well as patients who went to surgery due to PHPT in our study. Previous studies of mild PHPT patients have conflicting findings regarding elevated C-IMT $[3,4]$ or non-elevated C-IMT $[9,11]$. In our study, C-IMT was similar to the control group in PHPT patients, but A-IMT was found to be significantly higher in PHPT patients.

In some of the C-IMT assessments conducted in PHPT patients, C-IMT was found to be increased in patients with CV risk factors (DM, HL, HT, obesity and smoking) but similar to the control group in patients without these risk factors [5, 9]. In very rare cases, there is information that C-IMT may increase even if there isn't a CV risk factors [3,4]. Patients with risk factors that could affect the IMT were not taken for this reason and very careful attention was paid to this issue. In patients with PHPT who did not have CV risk fac-

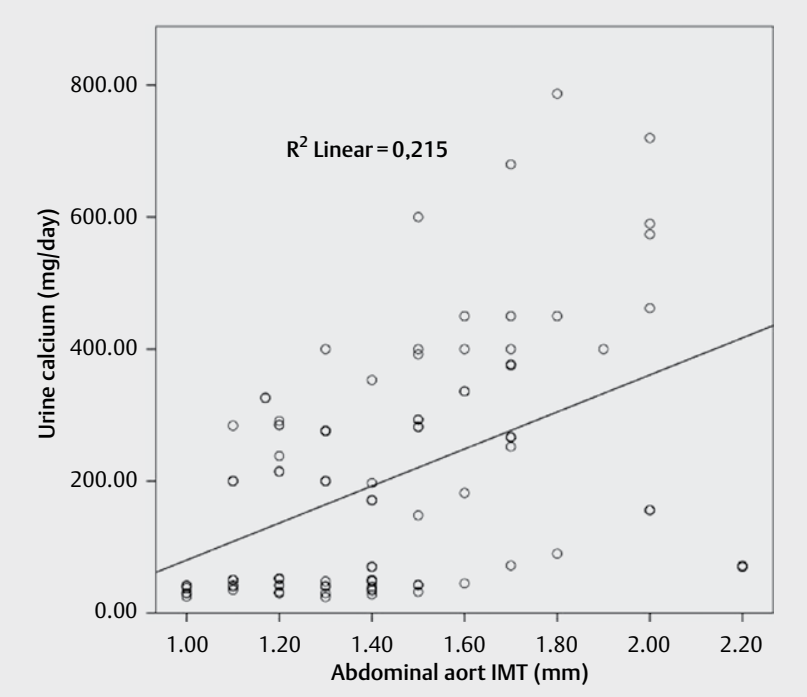

-Fig. 4 There was a significant correlation between urine calcium levels and abdominal aortic intima-media thickness (IMT) in patients with primary hyperparathyroidism.

tors, we found that C-IMT value was similar to the control group in accordance with most studies in the literature. However, this did not apply to A-IMT.

Two biochemical markers are important in PHPT patients for vascular involvement, which are elevated serum calcium and PTH levels. In studies with IMT increase, these two parameter increases were associated with IMT increase for physiopathology. However, the relationship between C-IMT and serum calcium and PTH levels in PHPT patients also has conflicting results. Some of these studies show a relationship between elevated serum calcium and PTH levels and C-IMT [18-21] but there are many conflicting studies on that matter. [3-5, 7, 9, 10]. Increased levels of calcium and PTH levels have been shown to negatively affect vascular function, especially by impairing vasodilatation and increasing vessel stiffness. $[7,8,22]$. In the study conducted by Lumachi et al. [9], it was reported that there is no relation between serum calcium and PTH levels and C-IMT and therefore serum calcium level is not correlated with carotid atherosclerosis development. The study by Luigi et al. [18] showed an independent relationship between urinary calcium level and C-IMT. In our study, both serum and urinary calcium levels and A-IMT increase were closely and independently related to each other. PTH levels were associated with A-IMT but this relationship was not independent. Our study supports the findings of the study conducted by Luigi et al. In addition, urinary calcium level and A-IMT value in our study was found to be independent determination of who underwent surgery, as well as serum phosphorus and PTH levels. These two findings suggested that serum calcium as well as urine calcium may be important for following PHPT patients.

In the literature, there is no data on abdominal-IMT increase or variability in PHPT patients, recently, Pep et al. [23], for the first time, found that the frequency and severity of abdominal aortic 
calcification with lateral abdominal radiographic thinning in the postmenopausal PHPT patients was greater in the control group. It was also reported that serum PTH level correlated with calcification in the same study and this finding could be correlated with CV risk in PHPT patients. In our study, a different and more objective assessment of vascular involvement, the abdominal-IMT assessment was performed in the same anatomic region,and higher A-IMT was detected in PHPT patients, similar to the previous study. However, in our study, the relationship between increased A-IMT and other CV risk factors in PHPT patients was not assessed, patients with risk factors were excluded because they had an effect on the A-IMT value.

In PHPT patients, matrix metallopeptidase- 9 is upregulated, and in addition to that, PTH enhances the genes in fat tissue and inflammation [24]. Chronic hypercalcemia has been shown to stimulate calcium accumulation in the IMT layer of the artery [19], and increased levels of PTH enhance collagen superimposition and re-organization in smooth muscle cells [25], in addition to increased production of atherosclerotic parameters from endothelial cells [26]. These changes lead to an increase in HT and atherosclerotic process in patients with PHPT. Atherosclerosis begins to develop as fatty streaks in childhood and can be detected early by sensitive imaging methods [27]. Because atherosclerosis develops first in the distal abdominal aorta, A-IMT measurement with new highresolution USG devices in adult patients can be used to detect early atherosclerosis development [12]. We hypothesized that increased serum calcium and PTH levels in patients with PHPT may show an earlier increase in abdominal A-IMT than C-IMT, and thus it is maybe more useful to use A-IMT instead of C-IMT. For this reason, we received A-IMT measurements from the abdominal aorta of our patients. In our study, histopathological vascular tissue examination was not carried out, but physiopathologic changes described in patients with PHPT were clearly explained. An increase in A-IMT in patients with PHPT is more prominent and significant than an increase in C-IMT. In addition, serum and urinary calcium levels are closely and independently associated with A-IMT.

There are some important limitations in this study. First of all, the number of patients gathered for the study is limited. Patients were not followed up and there was no data about efficacy in the effect of the treatment on the IMT. In addition, since the presentation of the association of A-IMT in patients with PHPT is the first of its kind in the literature, it is necessary to support the existing data with a study with more participants.

In our study only 8 patients has renal stone history, there is no skeletal fragility, coronary artery disease, stroke, and other advanced stage PHPT organ involvement. In our study, the reason for the lower renal stone in the PHPT patient group compared to the previous studies was thought to be due to the relatively younger patient group without cardiovascular risk factors. Therefore, A-IMT was not evaluated in this group of patients. Patients with CV risk factors are not included in the study in order to clearly visualize the effect of PHPT patients on IMT. For this reason, it is necessary to carry out studies on patients with these risk factors. It has been reported that CV mortality and morbidity are high in patients with PHPT and high PTH [28]. However, our study did not evaluate the prognosis of the disease.

\section{Conclusions}

While C-IMT is not significantly increased in PHPT patients, A-IMT is increased and A-IMT is more useful than C-IMT in showing vascular organ involvement in relatively early years of PHPT. A part of the renal USG, a routine examination for nephrolithiasis in PHPT patients, should be the A-IMT measurement, which is a very close neighbor, and the A-IMT value should be reported in the conclusion part of the renal USG. Measurement of A-IMT is a cheap, easy, reproducible and noninvasive parameter that can be used to detect IMT increase, which is an early finding of vascular involvement in patients with PHPT .

\section{Conflict of Interest}

The authors declare that they have no conflict of interest.

\section{References}

[1] Silverberg SJ, Walker MD, Bilezikian JP. Asymptomatic primary hyperparathyroidism. J Clin Densitom 2013; 16: 14-21

[2] Montalcini T, Terracciano R, Romeo S et al. Postmenopausal women with carotid atherosclerosis: Potential role of the serum calcium levels. Nutr Metab Cardiovasc Dis 2013; 23: 1141-1146

[3] Nuzzo V, Tauchmanovà L, Fonderico F et al. Increased intima-media thickness of the carotid artery wall, normal blood pressure profile and normal left ventricular mass in subjects with primary hyperparathyroidism. Eur J Endocrinol 2002; 147: 453-459

[4] Walker MD, Fleischer ], Rundek T et al. Carotid vascular abnormalities in primary hyperparathyroidism. J Clin Endocrinol Metab 2009; 94: 3849-3856

[5] Fallo F, Camporese G, Capitelli E et al. Ultrasound evaluation of carotid artery in primary hyperparathyroidism. J Clin Endocrinol Metab 2003; 88: 2096-2099

[6] Cansu GB, Yılmaz N, Özdem S et al. Parathyroidectomy in asymptomatic primary hyperparathyroidism reduces carotid intima-media thickness and arterial stiffness. Clin Endocrinol (Oxf) 2016; 84: 39-47

[7] Kosch M, Hausberg M, Vormbrock K et al. Studies on flow-mediated vasodilation and intima-media thickness of the brachial artery in patients with primary hyperparathyroidism. Am J Hyperten 2000; 13: 759-764

[8] Stamatelopoulos K, Athanasouli F, Pappa T et al. Hemodynamic markers and subclinical atherosclerosis in postmenopausal women with primary hyperparathyroidism. J Clin Endocrinol Metab 2014; 99 : 2704-2711

[9] Lumachi F, Ermani M, Frego M et al. Intima-media thickness measurement of the carotid artery in patients with primary hyperparathyroidism. A prospective case-control study and long-term follow-up. In Vivo 2006; 20: 887-890

[10] Ring M, Farahnak P, Gustavsson T et al. Arterial structure and function in mild primary hyperparathyroidism is not directly related to parathyroid hormone, calcium, or vitamin D. PLoS One 2012; 7: e39519

[11] Kosch M, Hausberg M, Barenbrock M et al. Arterial distensibility and pulse wave velocity in patients with primary hyperparathyroidism before and after parathyroidectomy. Clin Nephrol 2001; 55: 303-308

[12] Jarvisalo M], Jartti L, Nanto-Salonen K et al. Increased aortic intimamedia thickness: A marker of preclinical atherosclerosis in high-risk children. Circulation 2001; 104: 2943-2947 
[13] Bilezikian JP, Brandi ML, Eastell R et al. Guidelines for the management of asymptomatic primary hyperparathyroidism: Summary statement from the fourth international workshop. J Clin Endocrinol Metab 2014; 99: 3561-3569

[14] Catapano AL, Graham I, De Backer G et al. ESC Scientific Document Group 2016 ESC/EAS Guidelines for the Management of Dyslipidaemias. Eur Heart J 2016; 37: 2999-3058

[15] Mancia G, Fagard R, Narkiewicz K et al. 2013; ESH/ESC Guidelines for the management of arterial hypertension: The Task Force for the management of arterial hypertension of the European Society of Hypertension (ESH) and of the European Society of Cardiology (ESC). J Hypertens. 2013; 31: 1281-1357

[16] Bushinsky DA, Monk RD. Electrolyte quintet: Calcium. Lancet 1998; 352: 306-311

[17] Silverberg SJ, Shane E, Jacobs TP et al. A 10-year prospective study of primary hyperparathyroidism with or without parathyroid surgery. New England Journal of Medicine 1999; 341: 1249-1255

[18] Luigi P, Chiara FM, Laura Z et al. Arterial hypertension, metabolic syndrome and subclinical cardiovascular organ damage in patients with asymptomatic primary hyperparathyroidism before and after parathyroidectomy: preliminary results. International Journal of Endocrinology 2012; 408295

[19] Roberts WC, Waller BF. Effect of chronic hypercalcemia on the heart. An analysis of 18 necropsy patients. American Journal of Medicine 1981; 71: 371-384

[20] Rubin MR, Rundek T, McMahon DJ et al. Carotid artery plaque thickness is associated with increased serum calcium levels: The Northern Manhattan study. Atherosclerosis 2007; 194: 426-432
[21] Choi HS, Kim SH, Rhee Y et al. Serum parathyroid hormone is associated with carotid intima-media thickness in postmenopausal women. Int J Clin Pract 2008; 62: 1352-1357

[22] Colak S, Aydogan BI, Gokcay CA et al. Is primary hyperparathyroidism a cause of endothelial dysfunction? Clin Endocrinol (Oxf) 2017; 87: 459-465

[23] Pepe J, Diacinti D, Fratini E et al. High prevalence of abdominal aortic calcification in patients with primary hyperparathyroidism as evaluated by Kauppila score. Eur J Endocrinol 2016; 175: 95-100

[24] Christensen MH, Dankel SN, Nordbo Y et al. Primary hyperparathyroidism influences the expression of inflammatory and metabolic genes in adipose tissue. PLoS One 2011; 6: e20481

[25] Perkovic V, Hewitson TD, Kelynack KJ et al. Parathyroid hormone has a prosclerotic effect on vascular smooth muscle cells. Kidney and Blood Pressure Research 2003; 26: 27-33

[26] Rashid G, Bernheim J, Green J et al. Parathyroid hormone stimulates endothelial expression of atherosclerotic parameters through protein kinase pathways. American Journal of Physiology. Renal Physiology 2007; 292: F1215-F1218

[27] Berenson G, Srinivasan S, Bao W et al. Association between multiple cardiovascular risk factors and atherosclerosis in children and young adults. New Eng J Med 1998; 338: 1650-1655

[28] Vestergaard P, Mollerup CL, Frokjaer VG et al. Cardiovascular events before and after surgery for primary hyperparathyroidism. World J Surg 2003; 27: 216-222 